\title{
Prime Field Decompositions and Infinitely Divisible States on Borchers' Tensor Algebra
}

\author{
Gerhard C. Hegerfeldt \\ Institut fur Theoretische Physik, \\ Universität Göttingen, D-3400 Göttingen, Federal Republic of Germany * and \\ Université d'Aix-Marseille II, Luminy, Marseille, \\ Centre de Physique Théorique, CNRS, Marseille, France
}

\begin{abstract}
We generalize some notions of probability theory and theory of group representations to field theory and to states on the Borchers algebra $\underline{\mathscr{}}$. It is shown that every field (relativistic and Euclidean,...) can be decomposed into a countable number of prime fields and an infinitely divisible field. In terms of states this means that every state on $\mathscr{Q}$ is a product of an infinitely divisible state and a countable number of prime states, and in this formulation it applies equally well to correlation functions of statistical mechanics and to moments of linear stochastic processes over $\mathscr{\mathscr { Q }}$ or $\mathscr{Q}$. Necessary and sufficient conditions for infinitely divisible states are given. It is shown that the fields of the $\phi_{2}^{4}$-theory are either prime or contain prime factors. Our results reduce the classification problem of Wightman and Euclidean fields to that of prime fields and infinitely divisible fields. It is pointed out that prime fields are relevant for a nontrivial scattering theory.
\end{abstract}

\section{Introduction and Main Theorem}

The motivation of this paper comes from quantum field theory although the results have a wider range of applications. Let us consider, for example, two relativistic scalar fields $\varphi_{1}(x)$ and $\varphi_{2}(x)$ in Hilbert spaces $\mathfrak{H}_{1}$ and $\mathfrak{H}_{2}$, respectively, satisfying all Wightman axioms, including uniqueness of the vacua $\Omega_{1}$ and $\Omega_{2}$. We can then form the field

$$
\varphi(x):=\varphi_{1}(x) \times \mathbb{1}_{2}+\mathbb{1}_{1} \times \varphi_{2}(x)
$$

and consider it in the subspace $\mathfrak{S}$ of $\mathfrak{H}_{1} \times \mathfrak{H}_{2}$ in which $\Omega_{2}=\Omega_{1} \times \Omega_{2}$ is cyclic. Then $\varphi$ again satisfies all Wightman axioms and is irreducible in $\mathfrak{H}$. Equation (1.1) is analogous to the Kronecker product of Lie algebras, and we write

$$
\varphi=\varphi_{1} s \varphi_{2} .
$$

It is natural now to ask the reverse question: Can a given Wightman field $\varphi$ be written as an s-product of other Wightman fields? Clearly a similar problem can be formulated for other classes of fields.

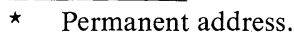


We first of all note that there are always trivial decompositions in which $\varphi_{1}=\varphi-F_{1}$, with ${ }^{1} F_{1} \in \mathscr{S}^{\prime}\left(\mathbb{R}^{4}\right), \mathfrak{H}_{1}=\mathfrak{H}, \Omega_{1}=\Omega$, and $\varphi_{2}=F_{1}, \mathfrak{H}_{2}=\mathbb{C}, \Omega_{2}=1$. These are of no interest.

To investigate the decomposition problem and to see that it is not restricted to quantum field theory we work with states on the tensor algebra $\mathscr{S}$ introduced by Borchers $[1]^{2}$ and studied in $[2,3]$. The elements of $\mathscr{S}$ are finite sequences of the form

$$
\underline{f}=\left(f_{0}, f_{1}, \ldots, f_{n}, 0, \ldots\right)
$$

with $f_{0} \in \mathbb{C}, f_{k} \in \mathscr{S}\left(\mathbb{R}^{d k}\right)$ where $d$ is the space-time dimension. We put $\mathscr{S}_{k}=\mathscr{S}\left(\mathbb{R}^{d k}\right)$. For $f \in \mathscr{S}_{n}$ and $g \in \mathscr{S}_{m}$ one defines

$$
\left(f^{*} \otimes g\right)\left(x_{1}, \ldots, x_{n+m}\right)=\bar{f}\left(x_{n}, \ldots, x_{1}\right) g\left(x_{n+1}, \ldots, x_{n+m}\right)
$$

and for $\underline{f}, \underline{g} \in \underline{\mathscr{P}}$ one defines $\underline{f}^{*} \times \underline{g}$ by

$$
\left(\underline{f}^{*} \times \underline{g}\right)_{N}=\sum_{n+m=N} f_{n}^{*} \otimes g_{m} .
$$

One calls $\underline{g}$ positive, $\underline{g \in \mathscr{S}^{+}}$, if $\underline{g}$ is a square or a sum of squares,

$$
\underline{g}=\sum \underline{f}_{i}^{*} \times \underline{f}_{i}
$$

$\mathscr{S}_{2 n}^{+}$is the set of all $g_{2 n} \in \mathscr{S}_{2 n}$ such that $\left(0, \ldots, g_{2 n}, 0 \ldots\right) \in \mathscr{\mathscr { S }}^{+}$. Linear functionals on $\mathscr{S}$ have the form

$$
T=\left(T_{0}, T_{1}, \ldots\right)
$$

with $T_{0} \in \mathbb{C}$ and $T_{k} \in \mathscr{S}^{\prime}$. $(\lambda, 0,0, \ldots)$ will be denoted by $\lambda . T$ is called positive if it is non-negative on $\mathscr{S}^{+} . T$ is a state, $T \in E(\mathscr{\mathscr { S }})$, if $T \geqq 0$ and $T_{0}=1$. We denote by $E_{1}(\mathscr{Q})$ the set of all states $T$ with $T_{1}=0$. The $n$-point functions of quantum fields, the correlation functions in statistical mechanics and the moments of linear stochastic processes over $\mathscr{S}_{1}$ are components of states.

The $s$-product of linear functionals $T^{(1)}, T^{(2)}$ is defined ${ }^{3}$ by

$$
\left(T^{(1)} s T^{(2)}\right)_{N}=\sum_{n+m=N} \sum_{P_{n m}} T_{n}^{(1)}\left(x_{i_{1}}, \ldots, x_{i_{n}}\right) \otimes T_{m}^{(2)}\left(x_{j_{1}}, \ldots, x_{j_{m}}\right)
$$

where $P_{n m}$ denotes a partition of $\{1, \ldots, n+m\}$ into two complementary ordered sets $\left\{i_{1}<\ldots<i_{n}\right\},\left\{j_{1}<\ldots<j_{m}\right\}$. The $s$-product is commutative ("symmetric"), distributive, associative and continuous in both factors simultaneously. With it one can define power series, in particular

$$
e^{T}:=\sum_{n} n !^{-1} T^{n}, \quad T^{n}=T S \ldots S T
$$

Translational invariance would imply $F_{1}=$ const.

The algebra $\mathscr{D}$ was independently introduced by Uhlmann [22].

Cf. Ruelle [4], p. 86, Borchers [2]. We use the notation of Borchers. 
which converges for every $T \in \mathscr{Q}^{\prime}$ [2]. If $T_{0}=1$ one can define the truncated functional $T^{t}$ by ${ }^{4}$

$$
T^{t}:=\sum(-1)^{n-1} n^{-1}(T-1)^{n}=\log T .
$$

This converges since in this case only a finite number of terms contribute for given $\underline{f} \in \mathscr{Q}$. We note that $T_{0}^{t}=0$, and if $T_{1}=0$ then $T_{2}^{t}=T_{2}$. Conversely, if $T_{0}=1$ then $^{5}$

$$
T=e^{T^{t}} \text {. }
$$

If $T_{0}^{(1)}=T_{0}^{(2)}=1$ then, by commutativity of the s-product,

$$
\left(T^{(1)} S T^{(2)}\right)^{t}=T^{(1) t}+T^{(2) t} .
$$

It follows from Eq. (1.4), [2] that if $\varphi_{1}, \varphi_{2}$ correspond to states $T^{(1)}, T^{(2)}$, then $\varphi_{1} s \varphi_{2}$ corresponds to $T^{(1)} s T^{(2)}$, and conversely. Thus we are dealing with a decomposition of a state as an $s$-product of other states. The above trivial decomposition of a field corresponds to the trivial decomposition $T=T^{(1)} S T^{(2)}$ where

$$
\begin{aligned}
& T^{(1)}=T-\left(0, F_{1}, F_{1} \otimes F_{1}, \ldots\right), \\
& T^{(2)}=\left(1, F_{1}, F_{1} \otimes F_{2}, \ldots\right) .
\end{aligned}
$$

States of the form (1.10) are called characters [2]; they give rise to 1-dimensional representations.

Definition 1.1. A state $T$ on $\mathscr{L}$ is called a prime state if it has no nontrivial decomposition of an s-product of other states and is not a character.

Definition 1.2. $T$ is a Wightman prime state if it has no nontrivial decomposition as an s-product of other Wightman states and is not a character. The corresponding fields are called Wightman prime fields. Similarly for Euclidean and other classes of states (fields).

As in probability theory [5] and group theory $[6,7]$ one can introduce the notion of infinite divisibility.

Definition 1.3. A state $T$ on $\mathscr{Q}$ is called infinitely divisible if for each $n \in \mathbb{N}$ there is a state $T^{1 / n}$, the $n$-th root of $T$, such that

$$
T=\left(T^{1 / n}\right)^{n} \equiv T^{1 / n} S \ldots S T^{1 / n} .
$$

The corresponding fields will be called infinitely divisible fields.

By Eq. (1.8), the truncation of $T^{1 / n}$ is $n^{-1} T^{t}$. Therefore, if $T$ is relativistic, Euclidean or Abelian, then so is $T^{1 / n}$. We note that the free and generalized free fields are infinitely divisible. Necessary and sufficient criteria will be given in Section 2.

Our main result on prime factorizations is analogous to that of Khinchine in probability theory ${ }^{6}$.

\footnotetext{
In probability theory this corresponds to going from moments to cumulants.

Again, for given $f \in \mathscr{\mathscr { }}$ only a finite number of terms are non zero.

6 See [8], pp. 170-172.
} 
Main Theorem. Every state $T$ on $\mathscr{S}$ is an s-product of two states, one of which is either 1 or is the s-product of a finite or countably infinite number of prime states, while the other is infinitely divisible with no prime factors.

Corollary. An analogous result holds for classes of states or fields (e.g., relativistic or Euclidean fields).

Remarks. (i) An infinite s-product of states is defined as a weak limit. If one works directly with fields and Hilbert spaces one has to use von Neumann's incomplete tensor products.

(ii) $T$ contains no prime factor if there is no decomposition of the form $T=P S R$ with $P$ prime; this is not implied by infinite divisibility.

(iii) The separability of the underlying space $\mathscr{S}_{1}$ yields the countability of the number of factors in a decomposition. The decomposition will in general be non unique.

(iv) The theorem carries over to other tensor algebras, in particular to $\mathscr{D}$ and to (non completed) tensor algebras $\mathfrak{U}(E)$ where $E$ is a locally convex separable space and where the components of the elements of $\mathfrak{U}(E)$ are in $E \otimes \ldots \otimes E$, i.e., finite linear combinations of product elements.

The proof, which only uses positivity of $T$, will be given in Section 4 . In Section 3 we prove some auxiliary results on factorizations, in particular compactness of a certain set of factors. In Section 5 we will discuss the existence of prime fields and possible implications of the factorization for the $S$-matrix of a HaagRuelle scattering theory. A note on Fermi fields can also be found there.

\section{Infinitely Divisible States}

Proposition 2.1. The set of infinitely divisible states on $\mathscr{S}$ is weakly closed. It is closed under the operation of taking s-products.

Proof. A net $\left\{T^{(\alpha)}\right\}$ of states converges to a state, $T$, if and only if $T^{(\alpha) t}$ converges; and if so, $T^{(\alpha) t}$ converges to $T^{t}[2]$. Let $T^{(\alpha)}$ be infinitely divisible and $T^{(\alpha)} \rightarrow T$. Since $\left(T^{(\alpha) 1 / n}\right)^{t}=1 / n T^{(\alpha) t}$, it follows that $T^{(\alpha) 1 / n}$ converges to a state $\hat{T}^{(n)}$ with $\hat{T}^{(n) t}=1 / n T^{t}$. Hence $\hat{T}^{(n)}=T^{1 / n}$. The last statement follows from the commutativity of the s-product. QED.

The next result is the analog of De Finetti's theorem [8].

Proposition 2.2. A state $T$ on $\mathscr{S}$ is infinitely divisible if and only if there is a sequence $\left\{\alpha_{v}\right\}$ of positive numbers and a sequence $\left\{T^{(v)}\right\}$ of states on $\mathscr{S}$ such that

$$
T=\lim _{\nu \rightarrow \infty} e^{\alpha_{\nu}\left(T^{(v)}-1\right)} .
$$

If $T_{1}=0$, one can choose $T_{1}^{(v)}=0$.

Proof. To show sufficiency we note that, for each positive $p \in \mathbb{R}$,

$$
e^{p \alpha_{v}\left(T^{(v)}-1\right)} \equiv e^{-p \alpha_{v}} e^{p \alpha_{v} T^{(v)}}
$$

is a state. Indeed, the s-product of two positive functionals is again positive [2], so the last term on the r.h.s. is a convergent sum of positive functionals and hence positive. The zeroth component of the 1.h.s. is 1 and so it is a state. Thus $T$ is the limit of a sequence of infinitely divisible states, and Proposition 2.1 applies. 
Necessity. Let $T$ be infinitely divisible. We claim that

$$
T=\lim _{n \rightarrow \infty} e^{n\left(T^{1 / n}-1\right)} .
$$

Indeed, truncating the terms on the r.h.s. we get

$$
\begin{aligned}
n\left(T^{1 / n}-1\right) & =n\left(e^{T^{t} / n}-1\right) \\
& =T^{t}+n^{-1} \sum_{v=2}^{\infty}\left(v ! n^{v-2}\right)^{-1}\left(T^{t}\right)^{v},
\end{aligned}
$$

which converges to $T^{t}$ for $n \rightarrow \infty$ since for each $\underline{f} \in \mathscr{\mathscr { L }}$ only a finite number of terms are non zero. QED.

We now give another criterion for infinite divisibility, and in order to make its formulation analogous to a criterion in probability theory ${ }^{7}$ we introduce the following terminology.

Definition 2.1. A linear functional on $\mathscr{Q}$ is called conditionally positive if it is positive on positive elements in the subspace $\left\{g ; g_{0}=0\right\}^{8}$.

Clearly the set of conditionally positive functionals forms a closed convex cone. If one considers $f=\left(0, f_{1}, f_{2}, \ldots\right)$, then for $g=f^{*} \times f$ one has the usual positivity condition. This shows in particular that the even components, except the zeroth, of a conditionally positive functional are positive. Note that all functionals of the form $\left(T_{0}, T_{1}, 0, \ldots\right)$ are conditionally positive but not necessarily hermitian, while

$$
\left(0,0, T_{2}, 0, \ldots\right)
$$

with $T_{2}$ positive on $\mathscr{S}_{2}^{+}$, is conditionally positive and hermitean. The truncated functional of a free and generalized free field has this form (Gaussian case).

Theorem 2.1. A linear functional $T$ on $\mathscr{L}$ is an infinitely divisible state if and only if $T_{0}=1$ and the truncated functional $T^{t}$ is conditionally positive and hermitean.

Proof. The necessity follows immediately from Proposition 2.2 since in Eq. (2.1) the exponent as well as its limit are conditionally positive and hermitean. To prove sufficiency we use a result of Yngvason [10] which states that the topology of $\mathscr{Q}$ is generated by the family of semi-norms $\left\{p_{S}\right\}$ of the form

$$
p_{S}(\underline{f})=S\left(\underline{f}^{*} \times \underline{f}\right)^{1 / 2}
$$

where $S$ is a positive linear functional on $\mathscr{Q}$. Hence there is a positive $S$ such that $T^{t}$ is continuous with respect to $p_{S}$. Instead of $T^{t}$ we now consider the functional

$$
S^{(\lambda)}:=p T^{t}+\lambda S, \quad \lambda>0, p \geqq 0 .
$$

This is again hermitean and conditionally positive, and on the subspace $\left\{g ; g_{0}=0\right\}$ it defines a semi-norm

$$
q_{\lambda}(\underline{g})_{:}=S^{(\lambda)}\left(\underline{g}^{*} \times \underline{g}\right)^{1 / 2}
$$

with respect to which $S^{(\lambda)}(\underline{g})$ is continuous, i.e.,

$$
\left|S^{(\lambda)}(\underline{g})\right| \leqq c_{\lambda} q_{\lambda}(\underline{g}), \quad g_{0}=0,
$$

7 Cf. [9] and [7].

8 This subspace is an ideal in $\mathscr{\mathscr { C }}$. If $\underline{g} \in \underline{\mathscr{L}}^{+}$and $g_{0}=0$ then also $g_{1}=0$ since the lowest non vanishing component is even [2]. 
for some $c_{\lambda}>0$. Let $g_{0}=0$. Then

$$
\begin{aligned}
& S^{(\lambda)}\left((1+\underline{g})^{*} \times(1+\underline{g})\right) \\
& \quad=S^{(\lambda)}\left(\underline{g}^{*} \times \underline{g}\right)+S^{(\lambda)}\left(\underline{g}^{*}\right)+S^{(\lambda)}(\underline{g})+S^{(\lambda)}(1) \\
& \quad \geqq q_{\lambda}(\underline{g})^{2}-2 c_{\lambda} q_{\lambda}(\underline{g})+S^{(\lambda)}(1) \\
& \quad \geqq-K_{\lambda}
\end{aligned}
$$

for some $K_{\lambda} \geqq 0$ and all $\underline{g}$ with $g_{0}=0$. Hence, for arbitrary $\underline{f} \in \underline{\mathscr{P}}$,

$$
S^{(\lambda)}\left(f^{*} \times \underline{f}\right) \geqq-K_{\lambda}\left|f_{0}\right|^{2} .
$$

Thus, for $n>K_{\lambda}, 1+S^{(\lambda)} / n$ is positive on $\underline{\mathscr{S}}^{+}$, as well as any power of it, i.e.,

$$
\left(1+S^{(\lambda)} / n\right)^{n} \geqq 0 \text {. }
$$

For $n \rightarrow \infty$ this converges ${ }^{9}$ to $e^{S^{(\lambda)}}$ which is therefore also positive. Since now

$$
\lim _{\lambda \rightarrow 0} e^{S^{(\lambda)}}=e^{p T^{t}}
$$

the r.h.s. is positive for every $p \geqq 0$ and a state since $T_{0}^{t}=0$. QED.

Remark. It is natural to define $T^{p}$ for $p \geqq 0$ by $e^{p T^{t}}$ if $T$ is infinitely divisible. Theorem 2.1 carries over to all tensor algebras $\mathfrak{A}(E)$ over an arbitrary vector space $E$. Indeed, the result of Yngvason [10] used above also holds for tensor algebras over finite-dimensional vector spaces. Since any $x \in \mathfrak{A}(E)$ is contained in a subalgebra of this kind positivity on $x^{*} \times x$ follows as before. For representations of Lie algebras a result analogous to Theorem 2.1 has been obtained by Streater [24].

Corollary 2.1. A linear functional $T$ on $\mathscr{S}$ is conditionally positive and hermitean if and only if it can be represented as

$$
T=\lim _{v \rightarrow \infty} \alpha_{v}\left(T^{(v)}-1\right)+T_{0},
$$

where $\left\{T^{(v)}\right\}$ are states, $\alpha_{\nu} \geqq 0$ and $T_{0}$ is real. If $T_{1}=0$, one can choose $T_{1}^{(v)}=0$ for all $v$.

Proof. $T-T_{0}$ is hermitean, conditionally positive and has vanishing zeroth component. Thus $e^{T-T_{0}}$ is an infinitely divisible state, and Eq. (2.1) applies. QED.

We note that if a linear functional $T$ is conditionally positive and $T_{2 m}=0$ for some $m \geqq 1$, then $T_{n}=0$ for all $n>2$; this follows from Schwarz's inequality. If no $T_{2 m}$ vanishes, $m \geqq 1$, then it is easy to construct from $T$ other conditionally positive functionals in the following way.

Definition 2.2. A sequence $\mathscr{C}=\left(c_{0}, c_{1}, \ldots\right)$ of real numbers will be called conditionally positive if $\left(c_{2}, c_{3}, \ldots\right)$ is of positive type, i.e. a moment sequence.

It is easy to see that $\mathscr{C}$ is conditionally positive if and only if

$$
c_{n}=c_{2}^{\prime} \delta_{n 2}+\int x^{n} d \mu(x), \quad n \geqq 2,
$$

where $\mu$ is a positive (possibly nonfinite) measure on $\mathbb{R}$ such that $\mu\{0\}=0$ and $c_{2}^{\prime} \geqq 0$.

9 This is seen by dividing Eq. (2.7) by its first component and then going over to the truncated functional. 
Proposition 2.3. Let $\mathscr{C}$ be a conditionally positive sequence. If $T \in \mathscr{L}^{\prime}$ is conditionally positive and hermitean, then so is

$$
\mathscr{C} \cdot T_{:}=\left(c_{0} T_{0}, c_{1} T_{1}, \ldots\right)
$$

Proof. We can assume $T_{0}=T_{1}=0$. Using Eq. (2.9) we consider the moment sequence $\mathscr{C}^{(\varepsilon)}$,

$$
c_{n}^{(\varepsilon)}=\int_{|x|>\varepsilon} x^{n} d \mu(x), \quad n=0,1, \ldots
$$

Using Eq. (2.8) for $T$ with $T_{1}^{(v)}=0$ for all $v$, we find

$$
\mathscr{C} \cdot T=\lim _{\varepsilon \rightarrow 0} \lim _{v \rightarrow \infty} \alpha_{v}\left(\mathscr{C}^{(\varepsilon)} \cdot T^{(v)}-c_{0}^{(\varepsilon)}\right)+\left(0,0, c_{2}^{\prime} T_{2}, 0, \ldots\right) .
$$

Now, $\mathscr{C}^{(\varepsilon)} \cdot T^{(v)}$ is positive [2], and therefore $\mathscr{C} \cdot T$ is a limit of conditionally positive functionals. QED.

We close this section with a structural result which is the analog of a theorem of Khinchine ${ }^{6}$.

Theorem 2.2. A state on $\mathscr{S}$ which contains no prime factor is infinitely divisible.

The proof will be given in Section 4 . Note that if $T$ is a Wightman or Euclidean state and if it does not contain a prime state of that class then it contains no prime state at all. Thus Theorem 2.2 also holds for classes of states. Its converse, however, does not hold.

\section{Auxiliary Results on Factorizations}

We first introduce some notations and prove some auxiliary results some of which are of independent interest.

Definition 3.1. Let $T \in E(\mathscr{\mathscr { S }})$. Then $\mathscr{F}(T)$ denotes the family of all factors of $T$. If $T=S S R$ then $S$ and $R$ are called complementary factors of $T$. If $T_{1}=0$, then $\mathscr{F}_{1}(T):=\left\{S \in \mathscr{F}(T) ; S_{1}=0\right\}$.

Lemma 3.1. Let $\left\{S^{(\alpha)}\right\}$ and $\left\{R^{(\alpha)}\right\}$ be nets of complementary factors of a state $T$ on $\mathscr{L}$. Then $\left\{S^{(\alpha)}\right\}$ converges weakly if and only if $\left\{R^{(\alpha)}\right\}$ does. If so, their limits are complementary factors of $T$.

Proof. We have $T^{t}=S^{(\alpha) t}+R^{(\alpha) t}$. Convergence of $S^{(\alpha)}$ and $R^{(\alpha)}$ is equivalent to that of $S^{(\alpha) t}$ and $R^{(\alpha) t}$. If $S^{t}$ and $R^{t}$ are their limits one has $T^{t}=S^{t}+R^{t}$ and hence $T=S$ S $R$. QED.

The next result will play a central role in the sequel.

Proposition 3.1. Let $T \in E_{1}(\underline{\mathscr{P}})$, i.e., $T_{1}=0$. Then $\mathscr{F}_{1}(T)$ is bounded on bounded sets and weakly closed, and thus strongly compact.

Proof. We first show that $\mathscr{F}_{1}(T)$ is bounded. It suffices to show that, for each $m$, the set of $m$-th components is bounded. We use induction. The statement is true for $m=0$; assume that it holds for $m=2 n-2$. We will prove it for $m=2 n$, $2 n-1$. 
Let $S \in \mathscr{F}_{1}(T)$. There is an $R \in \mathscr{F}_{1}(T)$ such that $T=S_{S} R$. From Eq. (1.4) we obtain for $T_{m}$ an expression of the form

$$
T_{m}=S_{m}+R_{m}+\text { remainder }\left(S_{\alpha} \otimes R_{m-\alpha}\right),
$$

where the remainder is a finite sum, and $2 \leqq \alpha \leqq m-2$ since $S_{1}=R_{1}=0$. Let $B \subset \mathscr{S}_{m}$ be a bounded set. Since $\mathscr{S}_{m}$ is a Montel space, $B$ is precompact. Therefore [11], for each $\alpha$, there are sequences $\left\{g_{i}\right\} \subset \mathscr{S}_{\alpha},\left\{h_{i}\right\} \subset \mathscr{S}_{m-\alpha}$, converging to zero, such that for every $f \in B$ there is a sequence $\left\{\lambda_{i}\right\}$ of complex numbers, $\sum\left|\lambda_{i}\right|<1$, with

$$
f=\sum_{i} \lambda_{i} g_{i} \otimes h_{i} .
$$

Applying the induction hypothesis to the bounded sets $\left\{g_{i}\right\}$ and $\left\{h_{i}\right\}$ one finds, for some $c>0, c=c_{B}$,

$$
\left|S_{\alpha} \otimes R_{m-\alpha}(f)\right| \leqq c \sum\left|\lambda_{i}\right|<c .
$$

Hence the remainder in Eq. (3.1) is bounded on $B$ for $m=2 n, 2 n-1$, uniformly for $S \in \mathscr{F}_{1}(T)$.

Now let $m=2 n$ and consider a bounded set $B^{+} \subset \mathscr{S}_{2 n}^{+}$. Then $T_{2 n}, S_{2 n}$, and $R_{2 n}$ are positive on $B^{+}$, and $T_{2 n}$ is bounded on $B^{+}$. Hence, by the boundedness of the remainder, $S_{2 n}$ is bounded on $B^{+}$uniformly for $S \in \mathscr{F}_{1}(T)$. If now $B$ is an arbitrary bounded set in $\mathscr{S}_{2 n}$ then Eq. (3.2), with $\left\{g_{i}\right\},\left\{h_{i}\right\} \subset \mathscr{S}_{n}$, yields boundedness of $S_{2 n}$ on $B$ by Schwarz's inequality,

$$
\left|S_{2 n}(f)\right| \leqq \sum\left|\lambda_{i}\right| S_{2 n}\left(g_{i}^{*} \otimes g_{i}\right)^{1 / 2} S_{2 n}\left(h_{i}^{*} \otimes h_{i}\right)^{1 / 2} \leqq c,
$$

$S \in \mathscr{F}_{1}(T), f \in B$, for some $c=c(B)$, since $\left\{g_{i}^{*} \otimes g_{i}\right\}$ and $\left\{h_{i}^{*} \otimes h_{i}\right\}$ are bounded sets in $\mathscr{S}_{2 n}^{+}$.

For $m=2 n-1$ one now uses an analogous argument with $\left\{g_{i}\right\} \subset \mathscr{S}_{n-1}$ and $\left\{h_{i}\right\} \subset \mathscr{S}_{n}$. This proves that $\mathscr{F}_{1}(T)$ is weakly and thus strongly bounded.

Weak closure follows from Lemma 3.1, and thus also strong closure. Strong compactness then follows from the fact that $\mathscr{L}$ is a Montel space. QED.

Remark. The result does not hold for $\mathscr{F}(T)$ owing to the unboundedness of the first components. The result also holds for the tensor algebra $\mathscr{D}$. For a (noncompleted) tensor algebra $\mathfrak{U}(E)^{10}$ one can show boundedness of $\mathscr{F}_{1}(T)$ on fixed elements.

For repeated application we note the following simple lemma.

Lemma 3.2. Let $\left\{T^{(\alpha)}\right\}$ be a net of states which is bounded. If $T_{2}^{(\alpha)}\left(f^{*} \otimes f\right)^{1 / 2} \rightarrow 0$ for $f$ in a dense subset of $\mathscr{S}_{1}$, then $T^{(\alpha)} \rightarrow 1$.

Proof. By Schwarz's inequality and boundedness one has, for $m \neq 0$,

$$
\left|T_{m}^{(\alpha)}\left(f_{1} \otimes \ldots \otimes f_{m}\right)\right| \leqq c T_{2}^{(\alpha)}\left(f_{1}^{*} \otimes f_{1}\right)^{1 / 2}
$$

for some $c>0$. Hence $T^{(\alpha)} \rightarrow 1$ on a dense set. From boundedness it then follows that $T^{(\alpha)} \rightarrow 1$ weakly. QED.

For the next result we introduce the following terminology.

Definition 3.2. A sequence $\left\{T^{(v)}\right\}$ of states is called factor-increasing if $T^{(v)} \in$ $\mathscr{F}\left(T^{(v+1)}\right)$, and factor-decreasing if $T^{(v+1)} \in \mathscr{F}\left(T^{(v)}\right), v=1,2, \ldots$

$\overline{10} \mathfrak{U}(E)$ is defined in Section 1. 
Proposition 3.2. Let $\left\{T^{(v)}\right\}$ be a factor-decreasing or bounded factor-increasing sequence of states on $\mathscr{S}$, with $T_{1}^{(v)}=0$ for all $v$. Then $\left\{T^{(v)}\right\}$ converges weakly.

Proof. Let $\left\{T^{(v)}\right\} \subset E_{1}(\mathscr{\mathscr { S }})$ be factor-increasing and bounded. By precompactness it has a converging subsequence, $\left\{T^{\left(v_{i}\right)}\right\}$, converging to $T$, say. Suppose that some other subsequence, $\left\{T^{\left(n_{k}\right)}\right\}$, converges to some $T^{\prime}$. Then each $T^{\left(v_{i}\right)}$ is a factor of some $T^{\left(n_{k}\right)}$ and hence of $T^{\prime}$. By Lemma 3.1 it follows that $T$ is a factor of $T^{\prime}$; analogously one has that $T^{\prime}$ is a factor of $T$, i.e., $T=T^{\prime}{ }_{S} R^{\prime}$ and $T^{\prime}=T$ s $R$ for some states $R, R^{\prime}$. This implies $T_{2}=T_{2}^{\prime}$ and thus $R_{2}=R_{2}^{\prime}=0$ since $T_{1}=T_{1}^{\prime}=0$. Hence $R=R^{\prime}=1$, by Lemma 3.2, and $T=T^{\prime}$, proving convergence. If $\left\{T^{(v)}\right\}$ is factordecreasing, then the sequence $\left\{R^{(v)}\right\}$ defined by $T^{(1)}=T^{(v)} S R^{(v)}$ is factor-increasing and bounded, by Proposition 3.1, and hence convergent since $R^{(v)} \in E_{1}(\mathscr{\mathscr { Q }})$ for all v. Lemma 3.1 then shows that also $\left\{T^{(v)}\right\}$ converges. QED.

We now consider a state $T \in E_{1}(\mathscr{\mathscr { S }})$ and introduce some notion of size of a factor $S \in \mathscr{F}_{1}(T)$. Let $\left\{h_{v}\right\}$ be a dense countable subset of $\mathscr{S}_{1}$, and let $\left\{\varrho_{v}\right\}$ be a sequence of positive numbers such that

$$
d_{0:}=\sum_{v} \varrho_{v} T_{2}\left(h_{v}^{*} \otimes h_{v}\right)<\infty .
$$

If $S \in \mathscr{F}_{1}(T)$, then $S_{2} \leqq T_{2}$, and thus

$$
d(S)_{:}=\sum_{v} \varrho_{v} S_{2}\left(h_{v}^{*} \otimes h_{v}\right) \leqq d_{0}<\infty .
$$

Lemma 3.3. Let $T \in E_{1}(\mathscr{\mathscr { S }})$, let $S \in \mathscr{F}_{1}(T)$ and let $d(S)$ be defined as in Eq. (3.5). If $S^{(i)} \in \mathscr{F}_{1}(T), i=1, \ldots, N \leqq \infty$, and if

$$
S=\left.\bar{S}\right|_{i} S^{(i)}
$$

then

$$
d(S)=\sum_{i} d\left(S^{(i)}\right) .
$$

If a net $\left\{S^{(\alpha)}\right\} \subset \mathscr{F}_{1}(T)$ converges to $S$, then $d\left(S^{(\alpha)}\right) \rightarrow d(S)$; the net converges to 1 if and only if $d\left(S^{(\alpha)}\right) \rightarrow 0$.

Proof. Since $S_{1}=S_{1}^{(i)}=0$ for all $i$, one has $S_{2}=\sum S_{2}^{(i)}$ and Eq. (3.6) follows immediately from Eq. (3.5). Now, if $d\left(S^{(\alpha)}\right) \rightarrow 0$ then $S_{2}^{(\alpha)}\left(h_{v}^{*} \otimes h_{v}\right) \rightarrow 0$ for each $v$, and hence $S^{(\alpha)} \rightarrow 1$, by Lemma 3.2. Conversely let $S_{2}^{(\alpha)} \rightarrow S_{2}$. Since $S_{2}^{(\alpha)} \leqq T_{2}$, a simple $\varepsilon / 2$-argument shows that $d\left(S^{(\alpha)}\right) \rightarrow d(S)$. QED.

\section{Proof of Theorem 2.2 and of Main Theorem}

We first turn to Theorem 2.2 and prove some preparatory lemmas.

Lemma 4.1. Let $T \in E_{1}(\mathscr{S})$ and let $T$ have no prime factor. Then, for each $\lambda$, $0 \leqq \lambda<1$, there exists some $S \in \mathscr{F}_{1}(T)$ with $d(S)=\lambda d_{0}$, where $d_{0}$ and $d(S)$ are given by Eqs. (3.4) and (3.5). As a consequence there are, for each $n \in \mathbb{N}$, states $S^{(n, v)} \in \mathscr{F}_{1}(T)$, $v=1, \ldots, n$, such that

$$
d\left(S^{(n, v)}\right)=d_{0} / n
$$

and

$$
T=\Gamma_{\mathrm{S}}^{n}{ }_{v=1}^{n} S^{(n, v)} .
$$


Proof. For each $\kappa, 0 \leqq \kappa<1$ we put

$\mu(\kappa)=\inf \left\{d(R) ; R \in \mathscr{F}_{1}(T), d(R)>\kappa d_{0}\right\}$.

Then there is a sequence $\left\{R^{(i, \kappa)}\right\}_{i=1}^{\infty} C \mathscr{F}_{1}(T)$ such that $\kappa d_{0}<d\left(R^{(i, \kappa)}\right)$ for each $i$ and $d\left(R^{(i, \kappa)}\right) \rightarrow \mu(\kappa)$ for $i \rightarrow \infty$. By going over to a subsequence, if necessary, we can assume, by Proposition 3.1, that $R^{(i, \kappa)}$ converges to some $R^{(\kappa)} \in \mathscr{F}_{1}(T)$ as $i \rightarrow \infty$. By Lemma 3.3, $d\left(R^{(\kappa)}\right)=\mu(\kappa)$. We first consider the case $\kappa=0$. We claim that $R^{(0)}=1$ so that a state without prime factors contains arbitrarily small factors. Indeed, if $R^{(0)} \neq 1$ there would exist $Q \in \mathscr{F}_{1}\left(R^{(0)}\right) \subset \mathscr{F}_{1}(T), Q \neq 1$, with $0<d(Q)<$ $d\left(R^{(0)}\right)$ and thus $\mu(0)$ would not be the above infimum.

Now let $\kappa=\lambda$. We claim that $d\left(R^{(\lambda)}\right)=\lambda d_{0}$. Indeed, assume $d\left(R^{(\lambda)}\right)=(\lambda+a) d_{0}$ with $a>0$. Since $R^{(\lambda)}$ contains arbitrarily small factors one can write $R^{(\lambda)}=R^{\prime} s R^{\prime \prime}$ with $0<d\left(R^{\prime \prime}\right)<a d_{0} / 2$. But then $\lambda d_{0}<d\left(R^{\prime}\right)<\mu(\lambda)$, a clear contradiction. Therefore we can choose $S=R^{(\lambda)}$.

Thus, for each $n \in \mathbb{N}$, there exist some $S^{(n, 1)} \in \mathscr{F}_{1}(T)$ with $d\left(S^{(n, 1)}\right)=d_{0} / n$. If $S^{(n, i)}$ has already been defined for $i \leqq v<n$ such that $d\left(S^{(n, i)}\right)=d_{0} / n$ and such that, for some $\hat{R}^{(v)} \in \mathscr{F}_{1}(T)$,

$$
T=\mathrm{S}_{i=1}^{v} S^{(n, i)} S \hat{R}^{(v)},
$$

then $d\left(\hat{R}^{(v)}\right)=(1-v / n) d_{0} \geqq d_{0} / n$. Therefore, there exists some $S^{(n, v+1)} \in \mathscr{F}_{1}\left(\hat{R}^{(v)}\right)$ with $d\left(S^{(n, v+1)}\right)=d_{0} / n$. If $v+1=n$ one has $S^{(n, n)}=\hat{R}^{(n-1)}$ and the construction terminates. QED.

Lemma 4.2. Let $T \in E_{1}(\mathscr{S})$ have no prime factor, and let $S^{(n, v)}$ be as in Lemma 4.1. Let $\left\{h_{\alpha}\right\} \subset \mathscr{S}_{1}$ be the functions used for $d_{0}$ in Eq. (3.4) and let $H$ be their linear hull. Then, on $\mathfrak{U}(H) \subset \mathscr{S}$ one has

$$
\lim _{n \rightarrow \infty} \sum_{v=1}^{n}\left(S^{(n, v)}-1\right)^{l}=0, \quad l=2,3, \ldots
$$

Proof. Since the first $2 l-1$ components of $\left(S^{(n, v)}-1\right)^{l}$ vanish, it suffices to prove convergence for elements of the form $f=\left(0, \ldots, 0, h_{\kappa_{1}} \otimes \ldots \otimes h_{\kappa_{m}}, 0, \ldots\right)$, $m \geqq 2 l$. Then, for finite $n$, the 1.h.s. of Eq. (4.3) is a finite sum of terms of the form

$$
\sum_{v=1}^{n} \prod_{i=1}^{l} S_{\alpha_{i}}^{(n, v)}\left(h_{i_{1}} \otimes \ldots \otimes h_{i_{\alpha_{1}}}\right)
$$

where, by Eq. (1.4), $\alpha_{i} \geqq 2, \sum \alpha_{i}=m$, and where the number of such terms depends on $l$ and $m$ only. Using Schwarz's inequality twice, $\left|S_{\alpha}\right| \leqq S_{2}^{1 / 2} S_{2(\alpha-1)}^{1 / 2}$ and $S_{2(\alpha-1)}^{1 / 2} \leqq$ $S_{2}^{1 / 4} S_{2(2(\alpha-1)-1)}^{1 / 4}$, each factor is estimated by

$$
c_{i} S_{2}^{(n, v)}\left(h_{i_{1}}^{*} \otimes h_{i_{1}}\right)^{1 / 2} S_{2}^{(n, v)}\left(h_{i_{\alpha_{i}}} \otimes h_{i_{\alpha_{i}}}^{*}\right)^{1 / 4}, \quad i=1, \ldots, l,
$$

where we have used boundedness, Proposition 3.1 .

For $i \geqq 3$ we estimate this by some constant independent of $n$ and $v$. Inserting this into Eq. (4.4) and applying the usual Schwarz's inequality to the sum over $v$, we obtain an estimate of the form

$$
\begin{gathered}
\left\{c^{\prime} \prod_{i=1}^{2} \sum_{v=1}^{n} S_{2}^{(n, v)}\left(h_{i_{\alpha_{1}}} \otimes h_{i_{\alpha_{i}}}^{*}\right)^{1 / 2} S_{2}^{(n, v)}\left(h_{i_{1}}^{*} \otimes h_{i_{1}}\right)\right\}^{1 / 2} \\
\leqq \\
\left.\quad \max _{j \leqq m} \varrho_{\kappa_{j}}^{-1} d_{0} / n\right\}^{1 / 4}\left\{c^{\prime} \prod_{i=1}^{2} T_{2}\left(h_{i_{1}}^{*} \otimes h_{i_{1}}\right)\right\}^{1 / 2}
\end{gathered}
$$

where we have used Eqs. (4.1) and (3.5). For $n \rightarrow \infty$ the last expression converges to zero which proves the lemma. 
Lemma 4.3. Let $T, S^{(n, v)}$, and $H \subset \mathscr{S}_{1}$ be as in the preceding lemma. Then, on $\mathfrak{U}(H) \subset \mathscr{L}$ one has

$$
T^{t}=\lim _{n \rightarrow \infty} \sum_{v=1}^{n}\left(S^{(n, v)}-1\right) .
$$

Proof. One has, for each $n$,

$$
T^{t}=\sum_{v=1}^{n} S^{(n, v) t}=\sum_{l=1}^{\infty} \sum_{v=1}^{n}(-1)^{l-1} l^{-1}\left(S^{(n, v)}-1\right)^{l} .
$$

If one applies the r.h.s. to some $f \in \mathscr{\mathscr { S }}$, only a finite number of terms in the sum over $l$ contributes. If $\underline{f} \in \mathfrak{U}(H)$ and if $n \rightarrow \infty$, only the term with $l=1$ remains, by Eq. (4.3). QED.

Proof of Theorem 2.2. After making a trivial decomposition of $T$, we can assume $T_{1}=0$. Then, by Eq. (4.5), $T^{t}$ is positive on $\left\{g \in \underline{\mathscr{P}}^{+} ; g_{0}=0\right\} \cap \mathfrak{U}(H)$. Since this set is dense in $\left\{g \in \mathscr{L}^{+} ; g_{0}=0\right\}$, it follows from the continuity of $T^{t}$ that $T^{t}$ is conditionally positive, and Theorem 2.1 applies. QED.

We now turn to the Main Theorem. We will denote by $\mathscr{P}(T)$ the family of all prime factors $P$ of $T$ and by $\mathscr{P}_{1}(T)$ those with $P_{1}=0$.

Proof of Main Theorem. After making a trivial decomposition we can assume $T_{1}=0$. We can also assume that $T$ is not prime and, by Theorem 2.2 , that $T$ contains prime factors. Let $d(S), S \in \mathscr{F}_{1}(T)$, be defined as in Eq. (3.5). By induction, we are going to construct a finite or infinite sequence of prime states $P^{(i)} \in \mathscr{P}_{1}(T)$ and states $R^{(i)} \in \mathscr{F}_{1}(T)$ such that, for each $k$,

$$
T=\Gamma_{i=1}^{k} P^{(i)} S R^{(k)}
$$

and such that either, in the finite case,

$$
\sup \left\{d(P) ; P \in \mathscr{P}_{1}\left(R^{\left(n_{0}\right)}\right)\right\}=0
$$

where $n_{0}$ is the highest index of the sequence, or, in the infinite case,

$$
\lim _{n \rightarrow \infty} \sup \left\{d(P) ; P \in \mathscr{P}_{1}\left(R^{(n)}\right)\right\}=0 .
$$

We choose some $P^{(1)} \in \mathscr{P}_{1}(T)$ with $d\left(P^{(1)}\right) \geqq d_{1} / 2$ where

$$
d_{1:}=\sup \left\{d(P) ; P \in \mathscr{P}_{1}(T)\right\} .
$$

We define $R^{(1)}$ by $T=P^{(1)} s R^{(1)}$. For induction, we now assume that $P^{(m)}$ and $R^{(m)}$ have been defined for $m \leqq n, n \geqq 1$. We then proceed as follows. Let

$$
d_{n+1:}=\sup \left\{d(P) ; P \in \mathscr{P}_{1}\left(R^{(n)}\right)\right\} .
$$

If $d_{n+1}=0$, we terminate the construction. Otherwise we select $P^{(n+1)} \in \mathscr{P}_{1}\left(R^{(n)}\right)$ with $d\left(P^{(n+1)}\right) \geqq d_{n+1} / 2$ and define $R^{(n+1)}$ by

$$
R^{(n)}=P^{(n+1)} s R^{(n+1)} .
$$

Then, Eq. (4.6) holds. If the construction does not terminate for a finite $n_{0}$ we have, for each $n$,

$$
d_{0} \geqq d\left(|\mathrm{~s}|_{i=1}^{n} P^{(i)}\right) \geqq \frac{1}{2} \sum_{i=1}^{n} d_{i} .
$$


Hence $d_{n} \rightarrow 0$ as $n \rightarrow \infty$ so that Eq. (4.8) holds. Furthermore, by Proposition 3.2, the limits

$$
\begin{aligned}
& S=\lim _{n \rightarrow \infty}|\mathrm{S}|_{i=1}^{n} P^{(i)} \\
& R=\lim _{n \rightarrow \infty} R^{(i)}
\end{aligned}
$$

exist and, by Lemma 3.1,

$$
T=S s R \text {. }
$$

We claim that $R$ or $R^{\left(n_{0}\right)}$, respectively, contains no prime factor. This is clear in the terminating case. In the infinite case assume that there exists some $P \in \mathscr{P}(R)$. We can assume $P_{1}=0$. By Proposition 3.2 and Lemma 3.1 we have, for each $n$,

$$
R^{(n)}=R s\left\lceil\mathrm{~S}_{i>n} P^{(i)} .\right.
$$

Hence $P \in \mathscr{P}_{1}\left(R^{(n)}\right)$ and thus $d(P) \leqq d_{n}$, for each $n$. Therefore $d(P)=0$ and $P=1$.

By Theorem 2.2, $R$ or $R^{\left(n_{0}\right)}$, respectively, are infinitely divisible. QED.

The corollary to the Main Theorem follows immediately if, in the above proof, one replaces the expression "prime factor" by "prime factor with respect to the class considered". The "new" prime factors only can become larger.

\section{Existence of Relativistic and Euclidean Prime Fields. Discussion}

The Main Theorem reduces the classification problem of Wightman fields to that of prime fields and infinitely divisible fields ${ }^{11}$. Classification of the latter seems not quite hopeless. There are strong indications that Wick products of free fields are again infinitely divisible. If so, one can construct new infinitely divisible fields from them by multiplying their truncated $n$-point functions by a conditionally positive sequence of real numbers (cf. Proposition 2.3). By analyticity the truncated functions can also be multiplied by the $n$-point functions of an arbitrary Wightman field $^{12}$. Nonrelativistic examples of such fields are well-known, e.g., the field of the ultralocal models $[12,13]$ are infinitely divisible.

Relativistic and Euclidean ${ }^{13}$ Prime Fields. Since the fields of the $\varphi_{2}^{4}$-theory [12, 13] are not generalized free fields they are either prime or contain prime fields.

\footnotetext{
11 In the theory of representations of simple compact Lie groups and Lie algebras one finds a similar situation. There every irreducible representation is obtained from the highest weight in the Kronecker product of fundamental representations. E.g., the irreducible representation $D_{j}$ of $\mathrm{SU}(2)$ can be obtained from the two-dimensional representation by restricting the $2 j$-fold Kronecker product to the symmetric subspace. Thus for $\mathrm{SU}(2)$ there is only a single "prime" representation.

12 This was suggested to me by H. J. Borchers.

13 In this context we mean by a Euclidean field an Abelian Euclidean covariant field whose $n$-point functions can be analytically continued to complex times to yield the $n$-point functions of a relativistic Wightman field $[14,15]$. It is conceivable that a Euclidean field is prime while the associated relativistic field is not, simply because the factors of the relativistic might only correspond to Euclidean Green's functions [16] but not to Euclidean fields.
} 
Indeed, the truncated 4-point function of the corresponding Euclidean field satisfies $[17,18]$

$$
S_{4}^{t}\left(x_{1}, \ldots, x_{4}\right) \leqq 0 .
$$

If the equality sign holds everywhere then the field is a generalized free field (Gaussian) by a general result of Borchers ${ }^{14}$. Since this is not the case the Euclidean field is not infinitely divisible, by Theorem 2.1 and Euclidean prime fields exist, by the Main Theorem. In this case neither the truncated relativistic 4-point function $\mathscr{W}_{4}^{t}$ can be positive since then one would have [20], for $\operatorname{Im}\left(z_{2}-z_{1}\right) \in V_{+}$,

$$
\mathscr{W}_{4}^{t}\left(\bar{z}_{1}, \bar{z}_{2}, z_{2}, z_{1}\right) \geqq 0
$$

which is in contradiction to Eq. $(5.1)^{15}$ unless the equality sign holds.

Unitarity of S-Matrix. It has been shown by Rinke [21] that if a field $\varphi$ describes a particle of mass $m>0$ and satisfies the assumption of the Haag-Ruelle theory, then the field $1 / \sqrt{2} \varphi s \varphi$ has a unitary $S$-matrix if and only if $\varphi$ has $S$ matrix $\mathbb{1}$, and it satisfies asymptotic completeness $\left(\mathfrak{H}_{\text {in }}=\mathfrak{H}=\mathfrak{H}_{\text {out }}\right)$ if and only if $\varphi$ is a free field. The factor $1 / \sqrt{2}$ merely normalizes the two-point function. The argument of [21] can be reversed to show that if a field $\psi$ satisfies the Haag-Ruelle assumptions with unitary $S$-matrix and if $\psi=1 / \sqrt{2} \varphi s \varphi$ for some Wightman field $\varphi$ then $\psi$ belongs to $S=\mathbb{1}$. This shows that infinitely divisible fields can only have a trivial scattering matrix ${ }^{16}$. The question under what conditions this reasoning can be carried over to other factorizations and under what conditions unitarity and nontriviality of the $S$-matrix are related to prime fields is presently under investigation.

Multicomponent Fields. The results of this paper all carry over unchanged to multiple-component fields, only the underlying space $\mathscr{S}_{1}=\mathscr{S}\left(\mathbb{R}^{d}\right)$ has to be changed to a space of vector-valued functions.

14 Lemma (H. J. Borchers, Vorlesungen über Quantenfeldtheorie, Göttingen 1966/67, unpublished): Let $A(x)$ be a Wightman field in a Hilbert space with a unique vacuum, and assume that the truncated Wightman function $W_{2 n}^{t}$ vanishes for one $n>1$. Then $A(x)$ is a generalized free field.

Sketch of Proof. One first shows that the vacuum expectation value of any $m$-fold commutator $K_{m}=\left[\ldots\left[A\left(x_{1}\right), A\left(x_{2}\right)\right] \ldots, A\left(x_{m}\right)\right]$ can be expressed by $W_{m}^{t}$. From this one gets $\left(K_{n}\left(x_{1}, \ldots, x_{n}\right) \Omega\right.$, $\left.K_{n}\left(y_{1}, \ldots, y_{n}\right) \Omega\right)-\left(K_{n}\left(y_{1}, \ldots, y_{n}\right) \Omega, K_{n}\left(x_{1}, \ldots, x_{n}\right) \Omega\right)=0$. Using the spectrum condition with respect to the centre of mass variables $\xi=1 / n \sum x_{v}, \eta=1 / n \sum y_{v}$ one finds that $\left(K_{n}\left(x_{1}, \ldots, x_{n}\right) \Omega, U(\eta-\xi) K_{n}\left(y_{1}, \ldots, y_{n}\right) \Omega\right)$ does not depend on $\eta-\xi$. The uniqueness of the vacuum then implies $K_{n}\left(x_{1}, \ldots, x_{n}\right) \Omega=$ $\Omega\left(\Omega, K_{n}\left(x_{1}, \ldots, x_{n}\right) \Omega\right)$. Local commutativity together with analyticity shows that $K_{n}\left(x_{1}, \ldots, x_{n}\right) A\left(z_{1}\right) \ldots$ $A\left(z_{m}\right) \Omega-A\left(z_{1}\right) \ldots A\left(z_{m}\right) \Omega\left(\Omega, K_{n} \Omega\right)$ vanishes identically in $z_{1}, \ldots, z_{m}$ which implies that $K_{n}$ is a $c$-number. Therefore $K_{n+1}, K_{n+2}, \ldots$ all vanish. Since either $n+1$ or $n+2$ is even one can repeat the argument which ends with $K_{2}$ being a $c$-number. Thus $A(x)$ is a generalized free field. QED.

By analytic continuation the same result holds for Euclidean Schwinger functions. A result in [19] is a special case of this.

15 For $z_{j}=\left(i x_{j}^{0}, x_{j}^{1}\right)$ and $z_{j} \neq z_{k}$ one has $S_{n}\left(x_{1}, \ldots, x_{n}\right)=\mathscr{W}_{n}\left(z_{1}, \ldots, z_{n}\right)$.

16 This is correct if one sticks to usual statistics. It was pointed out to me by $H$. Araki that this conclusion is possibly not valid in the parastatistics approach of Doplicher, Haag and Roberts [23]; it is tempting to speculate that infinite divisibility might be connected to infinite parastatistics. To settle this question one should try to classify infinitely divisible Wightman fields. 
Note Added in Proof. If $\psi_{1}$ and $\psi_{2}$ are Fermi fields then their s-product as defined in Eq. (1.1) will not satisfy the original anticommutation properties of $\psi_{1}$ and $\psi_{2}$. Therefore for Fermi fields the definition in Eq. (1.1) is not very appropriate. If the mapping $\psi_{2} \mapsto-\psi_{2}$ can be unitarily implemented, by $U_{\theta}$ say, then $\psi$ defined by

$$
\psi(x):=\psi_{1}(x) \times U_{\theta}+\mathbb{1}_{1} \times \psi_{2}(x)
$$

is again a Fermi field if $\psi_{1}$ and $\psi_{2}$ are. In particular, if the $n$-point functions of $\psi_{2}$ vanish for odd $n$ then $U_{\theta}$ exists and can be chosen to leave $\Omega_{2}$ invariant. In this case it is therefore natural to choose Eq. (5.3) for the definition of a modified s-product; in Eq. (1.4) for the functional there will appear minus signs. Truncation can again be defined by Eq. (1.6), but with the new s-product. It seems that all previous results carry over to this case. We note that for representations of canonical anticommutation relations (CAR) Eq. (5.3) has been used in [25] to define the concept of an infinitely divisible representation of the CAR, and it was shown that this class coincides with the quasi-free (Gaussian) representations; for more general anticommutation properties this is no longer true.

Acknowledgments. I would like to thank H. J. Borchers and J. Yngvason for stimulating discussions.

\section{References}

1. Borchers, H.J.: On the structure of the algebra of field operators. Nuovo Cimento 24, 214 (1962)

2. Borchers, H.J.: Algebraic aspects of Wightman field theory. In: Sen, R. N., Weil, C. (Eds.): Statistical mechanics and field theory. Haifa Lectures 1971. New York: Halsted Press 1972

3. Wyss, W.: On Wightman's theory of quantized fields. In: Lectures in theoretical physics. Boulder: University of Colorado 1968, New York: Gordon and Breach 1969

4. Ruelle, D.: Statistical mechanics. New York: Benjamin 1969

5. Loève, M.: Probability theory. Second edition. Princeton: van Nostrand 1960

6. Streater, R.F.: A continuum analogue of the lattice gas. Commun. math. Phys. 12, 226 (1969)

7. Guichardet,A.: Symmetric Hilbert spaces and related topics. Lecture Notes in Mathematics. (Chapter 4.) Berlin-Heidelberg-New York: Springer 1972

8. Lukacs, E.: Characteristic functions, 2nd ed. London: Griffin 1970

9. Gelfand,I.M., Vilenkin, N. Ya.: Generalized functions, Vol. 4, Chapter III, §4. New York: Academic Press 1964

10. Yngvason, J.: On the algebra of test functions for field operators. Commun. math. Phys. 34, 315 (1973)

11. Trèves, F.: Topological vector spaces, distributions, and kernels, cf. Theorem 45.2. New York: Academic Press 1967

12. Velo, G., Wightman, A. (Editors): Constructive quantum field theory. Lecture Notes in Physics 25. Berlin-Heidelberg-New York: Springer 1973

13. Simon, B.: The $P(\phi)_{2}$ Euclidean (quantum) field theory. Princeton: Princeton University Press 1974

14. Nelson,E.: Construction of quantum fields from Markoff fields. J. Funct. Anal. 12, 97 (1973)

15. Hegerfeldt, G.C.: From Euclidean to relativistic fields and on the notion of Markoff fields. Commun. math. Phys. 35, 155 (1974)

16. Osterwalder, K., Schrader, R.: Axioms for Euclidean Green's functions. II. Commun. math. Phys. 42, 281 (1975)

17. Lebowitz, J.: GHS and other inequalities. Commun. math. Phys. 35, 87 (1974)

18. Glimm, J., Jaffe, A., Spencer,T.: The particle structure of the weakly coupled $P(\varphi)_{2}$-model and other applications of high temperature expansions. In: Constructive quantum field theory. Lecture Notes in Physics 25, cf. p. 172. Berlin-Heidelberg-New York: Springer 1973

19. Newman, C.M.: Inequalities for Ising models and field theories which obey the Lee-Yangtheorem. Commun. math. Phys. 41, 1 (1975) 
20. Jost,R.: The general theory of quantized fields, p. 74. Providence: Am. Math. Soc. 1965

21. Rinke, M.: A remark on asymptotic completeness of local fields. Commun. math. Phys. 12, 324 (1969)

22. Uhlmann, A.: Über die Definition der Quantenfelder nach Wightman und Haag. Wiss. Z. KarlMarx-Univ. Leipzig, Math. Naturw. Reihe 11, 213 (1962)

23. Doplicher, S., Haag, R., Roberts, J.E.: Local observables and particle statistics. I. Commun. math. Phys. 23, 199 (1971)

24. Streater, R.F.: Infinitely divisible representations of Lie algebras. Z. Wahrscheinlichkeitstheorie verw. Geb. 19, 67-80 (1971)

25. Mathon,D., Streater,R.F.: Infinitely divisible representations of Clifford algebras. Z. Wahrscheinlichkeitstheorie verw. Geb. 20, 308-316 (1971)

Communicated by A. S. Wightman

Received June 10, 1975; in revised form July 17, 1975 
